\title{
Alternatives to Kronig-Kramers transformation and testing, and estimation of distributions
}

\author{
Bernard A. Boukamp a , J. Ross Macdonald ${ }^{\mathrm{b}}$ \\ - Department of Chemical Technology, Laboratory for Inorganic Chemistry, Materials Science and Catalysis, \\ University of Twente, P.O. Box 217, 7500 AE Enschede, The Netherlands \\ 'Department of Physics and Astronomy, University of North Carolina, Chapel Hill, NC 27599-3255, USA
}

Received 19 July 1994; accepted for publication 17 August 1994

\begin{abstract}
Two alternatives to Kronig-Kramers analysis of small-signal ac immittance data are discussed and illustrated using both synthetic and experimental data. The first, a derivative method of approximating imaginary-part response from real-part data, is found to be too approximate in regions where the imaginary-part varies appreciably with frequency. The second, a distribution of relaxation-times fitting method, is shown to be valuable for testing whether a data set satisfies the Kronig-Kramers relations and so is associated with a system whose properties are time-invariant. It also is valuable for estimating real- or imaginary-part response from the other part, usually with small error. Unlike Kronig-Kramers analysis, the second method usually requires no extrapolation outside the range of the measured data. Finally, this discrete-function method also allows one to estimate the distribution of relaxation times or activation energies associated with a given set of frequency-response data. This application is described and illustrated for both synthetic and experimental data and is shown to yield good but somewhat approximate results for the estimation of continuous distributions. It is particularly valuable for identifying response regions arising from a continuous distribution and distinguishing them from those associated with discrete time-constant response.
\end{abstract}

Keywords: Kronig-Kramers analysis; Electrochemical impedance spectroscopy (EIS)

\section{Introduction}

Analysis of small-signal ac frequency response data is a crucial step in gaining insight into the physical properties of the experimental material and system being investigated. It is also important in the use of such data for practical applications in the fields of corrosion, process monitoring, and surface-property evaluation. Such analysis is a part of impedance spectroscopy (IS) (more properly, immittance spectroscopy), a broad field which includes electrochemical impedance spectroscopy (EIS), and the investigation of the frequency response of semiconductor, dielectric, optical, and biological materials [1]. A list of acronyms and their definitions is included at the end of this work.

Here, we shall consider analysis methods of importance to all IS areas but will emphasize EIS response. In all these fields, analysis is usually carried out by fitting the data with complex nonlinear least squares (CNLS) to a mathematical response model or to an equivalent circuit which possibly includes one or more distributed circuit elements (DCE's), ones which cannot be described with a finite number of ideal circuit elements. Techniques and programs for such fitting are described in Refs. [2-5] and are used 
in the present work as appropriate. The latest versions of these computer programs are EQUIVALENT CIRCUIT, V. 4.51 [3] and LEVM, V. 6.1 $[4,5]$. But there are occasions when neither an appropriate mathematical model nor equivalent circuit are known for the experimental situation considered. It is then desirable to have available analysis techniques which still allow one to reach some useful conclusions about the experimental results even in the absence of such knowledge.

The Kronig-Kramers (KK) relations [6-8], which are coupled integral transforms satisfied by the real and imaginary parts of a complex function (here impedance) of a complex (frequency) variable, provide such an alternate analysis approach but suffer from some limitations. Although they have been applied in recent years to the analysis of EIS data [1,914], somewhat less attention has been devoted to their efficient application and limitations. One limitation is that they provide much less detailed information about specific response processes than do fitting with mathematical models or adequate equivalent circuits. When they can be readily applied, however, they allow one to assess whether the data are associated with a system having time-invariant properties and minimum-phase, passive response, since the KK relations (KKR) apply only for such systems. If system properties are slowly varying compared to the required measurement time, the KKR can still often apply to within the accuracy of the data, and even greater applicability is provided if one carries out a computer-controlled measurement from high to low frequencies and then back up to high frequencies. Averaging the results for the down and up measurements at equal frequencies then cancels linear time-variation effects.

Such tests for negligible time variation, which can be quite useful for corroding systems, require that one measure both parts of a complex immittance function (magnitude and phase or real and imaginary parts) over a wide frequency range and use one of the KKR with one of these quantities as an input to estimate the other. The estimate is then compared to the directly measured results for the corresponding quantity. It is usually desirable that this procedure be applied to both the real and imaginary parts of the data, requiring two integrations for each frequency for which the response is not expected to be negligibly small.

The KKR can be expressed in many forms $[1,8,15,16]$. The ones used herein, when applied to impedance data of the form $Z(\omega)=Z^{\prime}(\omega)+j Z^{\prime \prime}(\omega)$, are

$$
\begin{aligned}
& Z^{\prime}(\omega) \equiv Z^{\prime}(\infty)+(2 / \pi) \\
& \times \int_{0}^{\infty} \frac{\left[x Z^{\prime \prime}(x)-\omega Z^{\prime \prime}(\omega)\right] d x}{x^{2}-\omega^{2}}
\end{aligned}
$$

and

$$
Z^{\prime \prime}(\omega) \equiv-(2 \omega / \pi) \int_{0}^{\infty} \frac{\left[Z^{\prime}(x)-Z^{\prime}(\omega)\right] \mathrm{d} x}{x^{2}-\omega^{2}},
$$

where $R_{\infty} \equiv Z^{\prime}(\infty)$ and $\mathrm{j} \equiv \sqrt{-1}$. Here $R_{\infty}$ is the bulk or solution resistance of the material measured. Note that the first of these relations does not allow us to estimate $R_{\infty}$ from knowledge of $Z^{\prime \prime}(\omega)$, so its value must be determined separately. Further, since experimental data are measured over a limited frequency range, one must usually extrapolate the data beyond that range in order to carry out the above integrations sufficiently accurately.

Ways of carrying out these integrals for discrete data have been discussed recently in Ref. [15] (involving various transformed expressions for the KKR and the use of trapezoidal integration at geometric intervals in frequency) and Ref. [17] (involving polynomial or cubic spline fitting of the data and subsequent analytical integration ). The question of error transformation in the application of the KKR has been discussed in detail in Ref. [15] as well as integration errors, and reasonable ways of extrapolating finitefrequency-range data have been discussed in Ref. [17]. Finally, it is worth mentioning that in many areas of physics only a real or imaginary part of KKrelated data can be readily measured. Then, the appropriate $\mathrm{KK}$ relation is often used to produce an approximation for the other part of the data. For example, in optical measurements, one measures the reflectivity as a function of frequency and uses the KKR to calculate the associated phase. Then, one can calculate the real part of the index of refraction and the extinction coefficient. In solid state physics, this procedure is frequently employed to estimate the full complex dielectric function [16]. 
Consider now a general frequency-response relaxation function, $U_{i}(\Omega)$, which can represent either conductive-system dispersion $\left(i=\mathrm{C}\right.$, and $U_{\mathrm{C}}$ is an impedance $Z(\Omega)$ ), or dielectric-system dispersion ( $i=\mathrm{D}$, and $U_{\mathrm{D}}^{*}$ (the complex conjugate of $U_{\mathrm{D}}$ ) is a complex dielectric constant, $\left.\epsilon=\epsilon^{\prime}-\mathrm{j} \epsilon^{\prime \prime}\right)$. Here $\Omega \equiv \omega \tau_{0}$ is a normalized frequency involving the arbitrary relaxation-time quantity $\tau_{0}$. It should not be confused with the symbol for ohm $(\Omega)$. One can isolate the dispersive part of the response by defining the normalized immittance [18]

$$
\begin{aligned}
I_{i}(\Omega) & \equiv\left[U_{i}(\Omega)-U_{i}(\infty)\right] /\left[U_{i}(0)-U_{i}(\infty)\right] \\
= & I_{i}^{\prime}+\mathrm{j} I_{i}^{\prime \prime},
\end{aligned}
$$

and setting $U_{i}(0)$ and $U_{i}(\infty)$ equal to $R_{0}$ and $R_{\infty}$, respectively, for $i=\mathrm{C}$, and to $\epsilon_{\mathrm{D} 0}$ and $\epsilon_{\mathrm{D} \infty}$, respectively, for $i=\mathrm{D}$. Here $\epsilon_{\mathrm{D}_{\infty}}$ is the dielectric constant associated with the bulk capacitance of the filled measuring cell. With these definitions, $I_{i}(0)=1$ and $I_{i}(\infty)=0$. Although nearly all of the following work can be directly applied to dielectric dispersion, here we shall emphasize only dispersed conductive-system response. Then the complex modulus function associated with the impedance $Z(\omega), M(\omega)=$ $M^{\prime}(\omega)+\mathrm{j} M^{\prime \prime}(\omega)$, is just $\mathrm{j} \omega C_{\mathrm{V}} Z(\omega)$, where $C_{\mathrm{V}}$ is the capacitance of the empty measuring cell and involves the permittivity of vacuum, $\epsilon_{\mathrm{v}}$. But note that conducting-system dispersion and dielectric dispersion may occur simultaneously in the same general frequency region [19].

In this work, we shall consider in detail a derivative method of approximating imaginary-part response from the associated real-part data without extrapolation; a distribution-of-relaxation-times (DRT) alternative to the KKR which avoids many of their limitations; and the estimation of DRT's, or, equivalently, distributions of transition rates or activation energies (DAE's) [18], directly from IS frequencyresponse data. All KKR calculations herein were carried out with the EQUIVALENT CIRCUIT program $[3,17]$ and all DRT calculations with the LEVM program [4,5].

\section{An approximate transform using differentiation: exact and experimental data}

It has long been known [20] that given a portion of the real part of a complex response function which satisfies the KKR, say $U_{i}^{\prime}(\Omega)$, one can obtain an approximation over the same frequency region for the corresponding imaginary part, $U_{i}^{\prime \prime}(\Omega)$, using the relation

$U_{i}^{\prime \prime}(\Omega) \simeq-(\pi / 2)\left[\mathrm{d} U_{i}^{\prime}(\Omega) / \mathrm{d}\{\ln (\Omega)\}\right]$,

which will be applied here for $i=C$. A similar relation may be written with the $U$ 's replaced by normalized quantities. Since the result of the calculation is approximate even for exact data, it cannot be entirely consistent with the KK transformation from $U_{i}^{\prime}(\Omega)$ to $U_{i}^{\prime \prime}(\Omega)$, and thus it does not yield $U_{i}^{\prime \prime}(\Omega)$ exactly, but it has the virtue of not requiring any extrapolation of data beyond the measured frequency range, a process which may introduce errors of its own.

Before considering a more accurate alternative to the KKR, it is interesting to apply Eq. (4) to some exact and experimental data to illustrate its strengths and weaknesses. We shall begin by considering a thermally activated conducting system with an exponential distribution of activation energies or transition rates (EDAE) $[18,19,21]$, a physically-based model which has been found to yield frequency response results in good agreement with both experimental data and some empirical models such as that of Davidson and Cole [22]. For a thermally activated system, we may write

$\tau \equiv \tau_{\mathrm{a}} \exp (\mathscr{E})$,

where $\mathscr{E} \equiv E / k_{\mathrm{B}} T ; E$ is an activation energy (enthalpy); and $\left(\tau_{\mathrm{a}}\right)^{-1}$ is a barrier attempt frequency. We assume, as usual $[18,23]$, that given a random exponential distribution of barrier heights, the minimum $\mathscr{E}$ is 0 and the maximum is $\mathscr{E}_{\mathrm{H}}(<\infty)$; thus $\tau_{\mathrm{H}} \equiv \tau_{\mathrm{a}} \exp \left(\mathscr{E}_{\mathrm{H}}\right)$, and we shall here take $\tau_{0}=\tau_{\mathrm{H}}$, so $\Omega=\Omega_{\mathrm{H}}$.

As shown in previous work $[18,19]$, the normalized exponential distribution itself, $F(\mathscr{E})$, may be expressed in terms of $\phi(-\infty<\phi<\infty)$, where $\phi$ is a characteristic parameter of the distribution, as

$$
\begin{aligned}
& F(\mathscr{E})=\left[\phi /\left\{1-\exp \left(-\phi \mathscr{E}_{\mathbf{H}}\right)\right\}\right] \\
& \quad \times \exp \left[\phi\left(\mathscr{E}-\mathscr{E}_{\mathbf{H}}\right),\right.
\end{aligned}
$$


or, conveniently, for $\phi \leqslant 0$ as

$$
\begin{aligned}
& F(\mathscr{E})=\left[|\phi| /\left\{1-\exp \left(-|\phi| \mathscr{E}_{\mathbf{H}}\right)\right\}\right] \\
& \quad \times \exp (-|\phi| \mathscr{E})
\end{aligned}
$$

for $0 \leqslant \mathscr{E} \leqslant \mathscr{E}_{\mathrm{H}}$ and zero otherwise. For the usual conducting-system situation (Class I, see Ref. [19]), $\phi=1-k_{\mathrm{B}} T \eta$, and the temperature-independent quantity $\eta$ is a distribution-strength parameter. It follows that the normalized response function, $I_{\mathrm{C}}\left(\Omega_{\mathrm{H}}\right)$, hereafter written as $I(\Omega)$, may be expressed as

$$
\begin{gathered}
I(\Omega) \equiv \phi\left[1-\exp \left(-\phi \mathscr{E}_{\mathrm{H}}\right)\right]^{-1} \\
\times \int_{0}^{\sigma_{1}} \frac{\exp (-\phi x) \mathrm{d} x}{1+\mathrm{j} \Omega \exp (-x)} .
\end{gathered}
$$

This response is that of a DCE for any finite value of $\phi$. Although $I(\Omega)$ must be calculated by direct integration for arbitrary $\phi$, and it is included as one of the many distributed circuit elements available in the LEVM CNLS program, exact results are known for certain values of $\phi[18,24]$. Those for $\phi=1$ and $\phi=0$ are, respectively,

$$
\begin{aligned}
& I(\Omega)=\left[\left(1-r^{-1}\right)\right]^{-1} \\
& \quad \times \ln \left[(1+\mathrm{j} \Omega) /\left(1+\mathrm{j} \Omega r^{-1}\right)\right] /(\mathrm{j} \Omega),
\end{aligned}
$$

and

$I(\Omega)=1-x_{\overline{\mathbf{H}}}^{-1} \ln \left[(1+\mathrm{j} \Omega) /\left(1+\mathrm{j} \Omega r^{-1}\right)\right]$,

where $r \equiv \exp \left(x_{\mathrm{H}}\right) \equiv \tau_{\mathrm{H}} / \tau_{\mathrm{a}}$.

Fig. 1 shows an exact graph of $\ln \{F(\mathscr{E})\}$ versus $\mathscr{E}$ for $\mathscr{E}_{\mathrm{H}}=10$ and $\phi=0.7$. Note that the $\mathscr{E}$ scale is also a scale of $\ln \left(\tau / \tau_{\mathrm{a}}\right)=\ln \left(\tau / \tau_{0}\right)+\ln \left(\tau_{0} / \tau_{\mathrm{a}}\right)$. Thus, since the distribution is normalized, it may be considered as either a DAE or a DRT. For $\tau_{0}=\tau_{\mathrm{H}} \equiv \tau_{\max }$, it follows that $\ln \left(\tau / \tau_{\mathrm{H}}\right)=\ln \left(\tau / \tau_{0}\right)=\mathscr{E}-\mathscr{E}_{\mathrm{H}}$, and so $\tau_{\min } /$ $\tau_{0}=\exp \left(-\mathscr{E}_{\mathrm{H}}\right)=r^{-1}$. Thus, in general $\log _{10}\left(\tau_{\max } /\right.$ $\left.\tau_{\min }\right)=\mathscr{E}_{\mathrm{H}} \log _{10}(\mathrm{e})$. Also shown in Fig. 1 are $\log -\log$ schematic plots of the type of frequency response of $I_{i}(\Omega)$ and $\mathrm{j} \Omega I_{i}(\Omega)$ to be expected for $n=0.7$, and $\mathscr{E}_{\mathrm{H}}=10$. For $i=C$, the middle graphs show results of normalized impedance and the bottom ones show normalized modulus-level response, where $M \equiv \mathrm{j} \omega C_{\mathrm{V}} Z$. The curves also apply for the $i=\mathrm{D}$ dielectric case (middle set); then the bottom curves are
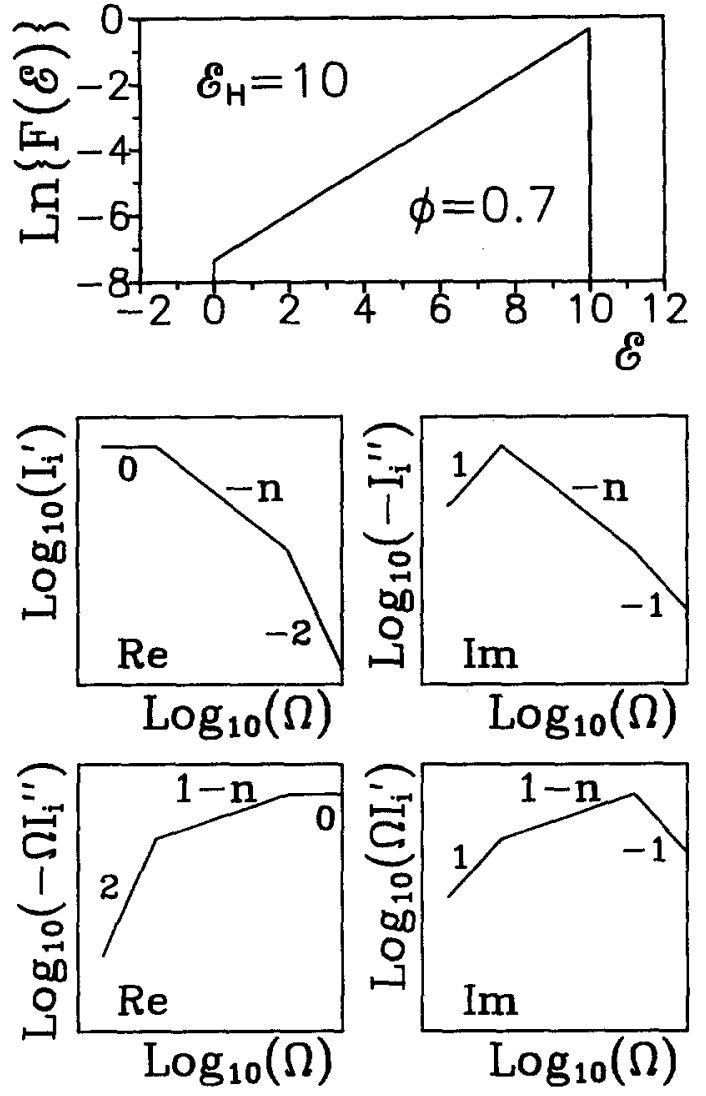

Fig. 1. Top: Exponential distribution of activation energies (EDAE) for $\phi=10$ and cutoffs at $\&=0$ and 10 . Bottom: plots showing the log-log frequency response schematically of $I_{i}(\Omega)$ and $\mathrm{j} \Omega I_{i}(\Omega)$ for the EDAE shown at the top. The quantity $\Omega$ is defined as $\omega \tau_{0}$. Here and elsewhere, unless otherwise noted, $\tau_{0}=1$ s. Ideal log-log slope values are shown for each section of the plots, and here $n \simeq \phi$. These results are pertinent for both conducting and dielectric systems for a wide range of $\phi$ values. See text for further discussion of $n$ values.

proportional to normalized admittance or conductivity. Note that for the dielectric case, $-I_{\mathrm{D}}^{\prime \prime}$ is related to $\epsilon^{\prime \prime}$ rather than to $-\epsilon^{\prime \prime}$.

All four schematic plots are included here to show explicitly the ideal, constant, log-log slopes of the various response regions, and, for clarity, all responses have been normalized to the same maximum value. The slopes not involving $n$ arise from the presence of high- and low- $\mathscr{E}$ cutoffs of $F(\mathscr{E})$, physically necessary for real-system response $[18,19,25]$. Although $n$ has been taken equal to $\phi$ for the present situation, these quantities are different entities and 
are not always even approximately equal $[18,19,21]$ since actual $\log -\log$ slopes of physically realizable real and imaginary responses must fall in the ranges $0 \leqslant\left|n_{\mathrm{Re}}\right| \leqslant 2$ and $0 \leqslant\left|n_{\mathrm{Im}}\right| \leqslant 1$. Finally, the larger $\mathscr{E}_{\mathrm{H}}$, the wider become the constant-slope middle regions.

It is worth mentioning that although the bottom four graphs are idealized and drawn specifically for $n=0.7$, for wide distributions they are appropriate for a large range of $\phi$ and $n$ values. Actual EDAE calculations show that the middle-region slopes shown on these graphs apply well for $0 \leqslant \phi \leqslant 1$, with $n \approx \phi$ and with closest agreement between $n$ and $\phi$ values near $\phi=0.5$. For the range $1<\phi \leqslant 1.3$, however, the actual $I^{\prime \prime} n$-value cannot exceed 1 , and, correspondingly, the actual slope, $1-n$, of the $\left|\Omega I^{\prime \prime}\right|$ real-part curve cannot be less than zero. But the other parts of the response show slopes in agreement with the predictions of Fig. 1 even for $\phi>1$. Similarly, for $-0.3 \leqslant \phi<0$, it is the $I^{\prime}$ and $\left|\Omega I^{\prime}\right|$ curves whose response disagrees with the predicted slopes on the corresponding curves, while the slopes of their pairs are as predicted. But note that these limitations do not preclude zero-slope behavior for the imaginary-part responses $\Omega I^{\prime}$ when $\phi=1$ or for the $\left|I^{\prime \prime}\right|$ response when $\phi=0$.

Next, Eqs. (8) and (9) were used to calculate highly accurate $Z(\Omega)$ and $M(\Omega)$ frequency-response conducting-system data for the two choices: (a) $\Delta R=10 \Omega, \mathscr{E}_{\mathrm{H}}=20$, and $\phi=1$, and (b) $\Delta R=1 \Omega$, $\mathscr{E}_{\mathrm{H}}=10$, and $\phi=0.7$. Here, $\tau_{0}$ was set to $1 \mathrm{~s}, R_{\infty}$ to zero, and, for convenience, $C_{\mathrm{V}}$ was taken as $1 \mathrm{~F}$. Then a generalized, automatic cross-validatory spline smoothing and differentiation program [26] was applied to the real parts of the data to obtain the derivatives in Eq. (4) and so to obtain estimates of the imaginary parts. Because of the accuracy of these synthetic data, no smoothing was needed for the quintic-spline fitting and differentiation. Fig. 2 shows the results of the approximate transform procedure, one which is known to work best for wide distributions. The solid curves show the exact responses and the dashed and dotted one show the results predicted by the differentiation method.

The top $\mathscr{E}_{\mathrm{H}}=20$ curves agree very closely in the middle flat region of $M^{\prime \prime}$; here $n=\phi$, so the slope $1-n$ is zero. But the exact and estimated curves begin to diverge at the corners and rapidly become quite different as $M^{\prime \prime}$ decreases. Remember that relatively small deviations on these $\log -\log$ plots correspond to

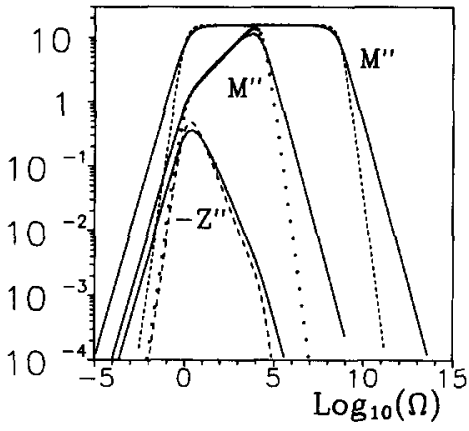

Fig. 2. Estimation of imaginary parts of a KK pair from their real parts by an approximate differentiation method. The solid lines show exact EDAE response results, and the other lines are estimated. The top curves are for $\Delta R=10 \Omega, \mathscr{E}_{\mathrm{H}}=20$, and $\phi=1$, while the others are for $\Delta R=1 \Omega, \mathscr{E}_{\mathrm{H}}=10$, and $\phi=0.7$. The units of $-Z^{\prime \prime}$ are ohms.

appreciable ones for $M^{n}$ itself. Generally, however, the differentiation method does a very respectable job for this wide-distribution situation if one is not interested in accurate small values.

The other curves in the figure are for $\mathscr{E}_{\mathrm{H}}=10$ and $\phi=0.7$ and should be compared to the corresponding schematic curves for Fig. 1. Here the estimates are worse, although the approximately constant-sloperegion of the $M^{\prime \prime}$ estimate is close to exact response. It is interesting that the low-frequency-region estimates of both $M^{\prime \prime}$ and $-Z^{\prime \prime}$ are closely the same (and not close to the exact responses). Spline log-log slope estimation using the original accurate data showed that $n(\Omega)$ was only close to $\phi=0.7$ for limited frequency regions. Let $d \equiv \Omega_{2} / \Omega_{1}$ denote the frequency range over which the agreement is of the order of $10 \%$. Then the spline calculations of $n(\Omega)$ yielded $d \simeq 100$ for $-n$ in the range -0.65 to -0.75 for $Z^{\prime}$ and $d \simeq 16$ for $-Z^{\prime \prime}$. For $(1-n)$ in the region 0.32 to $0.28, d$ was about 2.5 for $M^{\prime}$ and $\sim 10$ for $M^{\prime \prime}$.

Fig. 3 shows results for the transformation of $\mathrm{CaTiO}_{3}: \mathrm{Al}^{3+}$ data, kindly provided by Nowick [27], using the above methods. The data involved capacitance and dissipation factor values at 17 frequencies. Since the appropriate value of $C_{\mathrm{V}}$ was unavailable, the value $C_{\mathrm{v}}=1 \mathrm{pF}$ was used to transform to the epsilon immittance level; thus, absolute values in the figure are not fully meaningful. The $\epsilon^{\prime}$ data used for the transformation were given to seven significant figures, and although these values were certainly not as accurate as this might suggest, the data varied very 


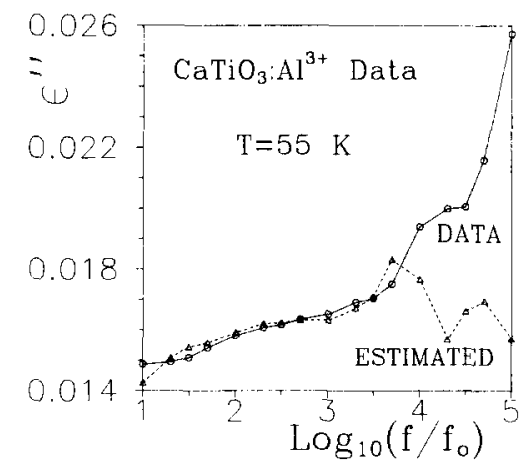

Fig. 3. Application of the differential-estimate method to experimental data for $\mathrm{CaTiO}_{3}$ doped with aluminum. Here and elsewhere, unless otherwise noted, $f_{0}=1 \mathrm{~Hz}$.

smoothly and regularly with frequency. The figure shows, nevertheless, that at the higher frequencies the estimated $\epsilon^{\prime \prime}$ values are quite irregular and agree poorly with the measured $\epsilon^{\prime \prime}$ values. Thus, at least for $f=10^{3}$ to $10^{5} \mathrm{~Hz}$, the differentiation method is inadequate for these data.

\section{The DRT alternative to Kronig-Kramers testing and transformation}

Fig. 4 shows two very general equivalent circuits for fitting data. The top one is essentially a Voigt circuit and the bottom one a Maxwell circuit [28]. Define the integer $M$ as the number of $R C$ pairs (e.g., $R_{1} C_{1}, R_{2} C_{2}$, etc.) actually used in the fitting. With the proper choices of circuit element values and with $M$ sufficiently large, one can fit virtually any data which are consistent with the KKR since the response of all such passive equivalent circuits satisfies the KKR. Nevertheless, the top circuit is physically more appropriate for a conductive system and the bottom one for dielectric response. Note that the $C_{\mathrm{P}}$ capacitance element may be used to represent the high-frequency-limiting capacitance, $C_{\mathrm{g}}$ or $C_{\infty}$, and $R_{0}$ in the top circuit may be used for the high-frequency-limiting bulk resistance, $R_{\mathrm{B}}$ or $R_{\infty}$.

Although fitting with a finite value of $M$ is entirely appropriate when the data are associated with a finite number of time constants (discrete response), the present method necessarily approximates continuous-response data, such as that arising from a DCE, by the combination of a finite number of discrete,

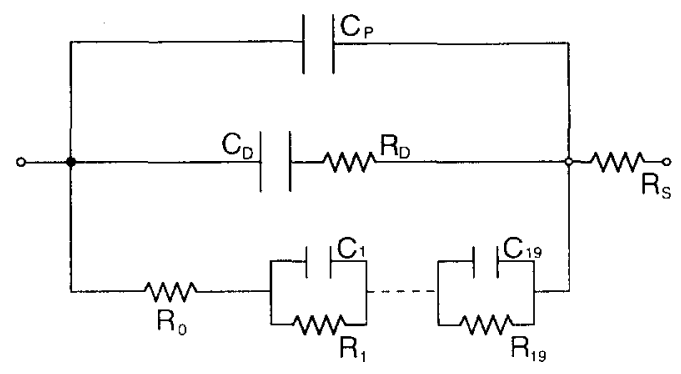

(a)

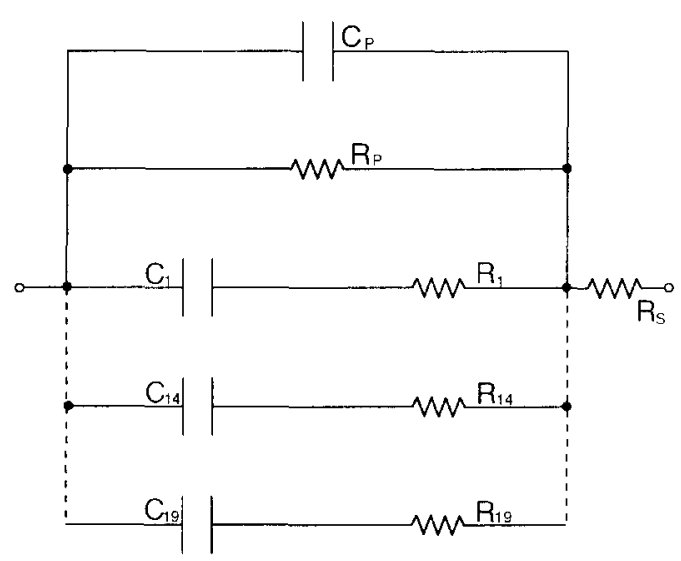

(b)

Fig. 4. Equivalent circuits used for DRT fitting of data. (a) Voigt circuit for a conductive system; (b) Maxwell circuit for a dielectric system.

single-time-constant responses. Nevertheless, even for data associated with a continuous distribution of relaxation times, usually a moderate value of $M$ allows one to fit typical experimental data to within its intrinsic random errors. But reasonably accurate representation of CPE response requires a minimum of several time constants, with more needed as the range of such response grows. It is worth mentioning that although the KKR are not useful if experimental data involve complete blocking behavior, the DRT approach may still be used.

By using one of the circuits of Fig. 4 for fitting, one does not need to have available a more detailed and possibly more physically appropriate circuit model in order to obtain an excellent fit of such data. Further, the results of fitting with one or the other of these circuits can be interpreted in terms of a discrete DRT or a discrete DAE $[8,19,29,30]$, as discussed later. 
The circuits are particularly valuable for testing whether the data satisfy the KKR because only a limited fit can be obtained if they do not (see later illustrations), while a fit restricted only by the accuracy of the data can be obtained using a sufficient number of $R C$ pairs if they do. An important advantage for usual experimental data, which covers only a limited frequency range, is that this approach to KKR verification does not require extrapolation of the measured data outside the measured frequency range, always a somewhat uncertain procedure.

Although the use of the top circuit (without the $C_{\mathrm{P}}$, $C_{\mathrm{D}}$, and $R_{\mathrm{D}}$ elements) has been recently usefully advocated for preliminary "measurement model" data fitting [31,32], these DRT or DAE circuits have been used for fitting for many years. But only relatively recently, with the availability of powerful CNLS fitting programs, has it become straightforward and practical to fit data to such equivalent circuits when $M$ is appreciable. The current version of the LEVM program can fit data with $M$ up to 19 . Here, where we deal with conductive-system data, we shall be primarily concerned with using the top circuit, although recent work [30] considers the application of the bottom one in detail.

Incidentally, when $M$ is of the order of 4 or less, one can usually carry out satisfactory DRT fitting by selecting fairly arbitrary best-guess parameters and then letting the fitting process iterate to full convergence. But for $M=5$ or more, there are ten or more free parameters to be estimated. To avoid the trap of getting caught in a local, but no absolute, minimum during the fitting, it has been found useful to first obtain a fit with small $M$, and then progress by separate fits for each increasing $M$ value, each one requiring two more initial parameter guesses. Thus, for $M=19$, one might have to carry out up to 17 or 18 separate fits. But each such individual fitting usually converges quickly, and only for synthetic data would one be concerned with so large a value of $M$. Although the fit convergence time for a given $M$ depends on the stringency of the fitting criteria used in LEVM and on how close free parameter guesses are to their converged values, the time required for a fit starting at $M$ and going to $M+1$ is roughly linear with $M$ and is closely linear when some correction for iterationnumber differences is included.

We shall apply the DRT fitting approach to three sets of IS experimental data which illustrate different possibilities and limitations of this approach and will compare them with those obtained with direct KKR testing. The complex-plane response at the impedance level of the first of these is shown in Fig. 5. These data, which involved the SOFC cathode, a porous lanthanum-doped strontium manganite mixed conductor, were kindly supplied by van Heuveln of the Energy Centre Netherlands, Petten [33]. All $Z$ data considered herein are in ohms, and we see that for the present response, that of a fuel-cell cathode, the impedance is very low. Since most of the open circles, the DRT-fit estimates obtained with $M=9$, surround the solid-symbol data points, a good DRT fit was obtained here, indicating consistency with the KKR.

But these data are unusual. First, of the nine parallel $R C$ pairs of the DRT fit, one $R$ and its associated $C$ were negative and three other $C$ 's were also negative, although for most data it is found that all $R$ 's and $C$ 's turn out to be positive. Note that although a negative resistance alone is inconsistent with passivity and thus with the KKR, when the zero-frequency limiting resistance of the total system is positive, as it is here, the KKR may still apply. The original measurements showed that for frequencies below about 1 $\mathrm{Hz},-Z^{\prime \prime}$ became very small and began to oscillate in sign irregularly and apparently rather randomly down to the lowest measured frequency, $0.05 \mathrm{~Hz}$. Now if the material and/or electrode properties of a measured system are varying with time at an approximately constant rate, one would expect that the result of such variation would be least apparent at high frequencies, where the change over a few periods might well be entirely negligible, and most influential at low

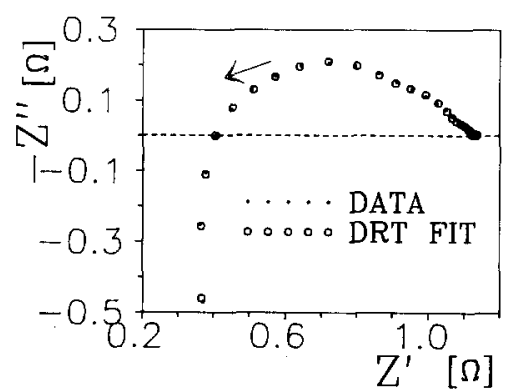

Fig. 5. Complex-plane impedance plot of data measured on a fuelcell cathode. The open circles show DRT fitting results with $M=9$, and the arrow indicates the direction of increasing frequency. 
frequencies, where the variation could be large during even a single period.

It seems highly likely that the present data set involves time-varying effects of this kind. It was, in fact, not found practical to carry out KKR testing on it because of the difficulty in obtaining a sensible lowfrequency extrapolation needed for the $\mathrm{KK}$ integrations. It was therefore decided to omit data values below $3.6 \mathrm{~Hz}$ before carrying out DRT fitting of the remainder of the data. The figure shows, however, that for the range covered, $3.64 \mathrm{~Hz}$ to $10^{6} \mathrm{~Hz}$, the effects of time variation were indeed negligible, and thus one can infer that over this range the data seem to satisfy all the criteria required for $\mathrm{KK}$ applicability. Incidentally, it was found that for this material in the steady state, only three $\tau$ 's were needed to achieve a good fit.

The second data set, kindly provided by Brinkman of the University of Twente [33], involved terbiadoped, yttria-stabilized zirconia with solid gold electrodes, used to suppress any oxygen-electrode reaction. Measurements were carried out in a nitrogen atmosphere with only traces of oxygen present. Thus, one might expect that the out-diffusion of oxygen during measurement could change the electronic conductivity of the material, resulting in time-varying behavior. The measured frequency response is shown in Fig. 6 by the solid points and lines. Here $Z^{*} \equiv Z^{\prime}-\mathrm{j} Z^{\prime \prime}$ is the complex conjugate of $Z$. For clarity, not all data points have been included in this and

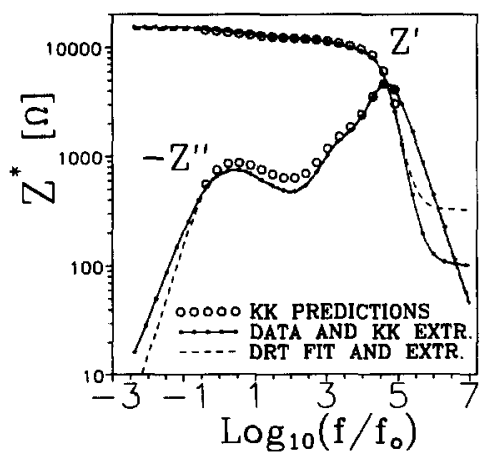

Fig. 6. $\mathrm{Log}-\log$ frequency response of the complex-conjugate impedance components of terbia-doped, yttria-stabilized zirconia with two evaporated gold electrodes. Here the open circles show $\mathrm{KK}$ transform results, and $f_{0}=1 \mathrm{~Hz}$ in this and all other figures. The DRT fit used $M=6 R C$ pairs. "Extr" stands for extrapolated data estimates. some of the other figures, although they were all used in the fittings. The open circles show KKR predictions and the dashed lines show results obtained from DRT fitting with $M=6$, here also including $R_{\infty}$ as a free fitting parameter.

In Fig. 6 "KK EXTR" stands for the high-and lowfrequency extrapolations needed for KK estimation. In contrast, after DRT fitting over the measured data range, the estimated fit parameters were used to extrapolate the DRT predictions at both ends of the range. As expected, they have limiting slopes in agreement with those of the middle pair of Fig. 1 (ignoring the effect on $Z^{\prime}$ of the $R_{\infty}$ value of about 320 $\Omega)$. Clearly, the DRT extrapolations, automatic consequences of the fitting, are appreciably different from those used in the KK transforms, except for those for $-Z$ " at high frequencies. These DRT extrapolations arise from the smallest and largest $\tau \equiv R C$ time constants estimated from the fit. If one defines the frequencies associated with them by the relation $2 \pi f \tau=1$, then for the present case the associated frequencies were about $0.72 \mathrm{~Hz}$ and $4.5 \times 10^{4} \mathrm{~Hz}$, values which lie about a factor of two away from the smallest and largest measured frequencies, here $0.4175 \mathrm{~Hz}$ and $10^{5}$ $\mathrm{Hz}$.

Although the present results show, as expected, that many of the KKR predictions are very poor, especially those at the lower frequencies, the DRT fitting yielded quite good results over the original data range. Let us define $S_{\mathrm{F}}$ as the standard deviation of the relative residuals of the fit, just the standard deviation of the fit itself for proportional weighting. The present fit yielded a value of $S_{\mathrm{F}}$ of about 0.027 , indicating that most relative residuals were below 3 or $4 \%$. But it was found that using an $M$ value greater than the relatively small value of 6 led to larger estimated parameter standard deviations and to very little further reduction in $S_{\mathrm{F}}$. This is itself an indication of possible problems with the data. For impedance data which satisfy the KKR, it is found that, as $M$ increases, $S_{\mathrm{F}}$ decreases by a factor of about 1.6 from a fit with $M$ to one with $M+1$. But this factor finally decreases and approaches unity as errors in the data begin to set a limit to the minimum $S_{\mathrm{F}}$ possible.

Fig. 7 shows the relative residuals of the DRT fit described above. To guide the eye, the relative residual values have been connected by straight lines. It is clear that those for the real part show strong system- 


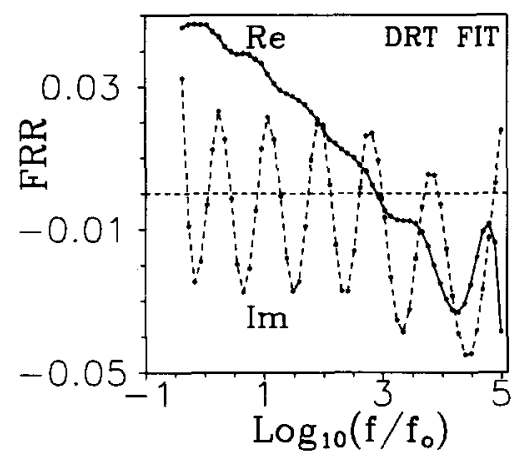

Fig. 7. Fit relative residuals (FRR) for the DRT fit of the data of Fig. 6.

atic behavior roughly consistent with time-varying effects, ones of most importance at low frequencies. The oscillatory systematic behavior, most evident for the imaginary-part results, are primarily a consequence of the small value of $M$ used here, but they too include a smaller linear component. Even with exact synthetic data associated with a continuous distribution such as that of a constant-phase element (CPE) [1], one finds such oscillations, ones with roughly $M$ equal-magnitude peaks, but with magnitudes decreasing rapidly as $M$ increases [24]. This behavior is a necessary consequence of the approximation of continuous response by a model involving a finite number of relaxation times when proportional weighting is used in the fitting as it was here [4,24,29].

To summarize: the present DRT results yield somewhat conflicting signals about the applicability of the KKR. The fit of the data is quite adequate when plotted on a log-log plot, but the real-part relative residuals, a much more sensitive indicator of discrepancies, show systematic behavior of time-varying character, as do the imaginary part ones to a lesser extent. Thus, it is reasonable to assume from the DRT fit alone that the KKR are inapplicable or, at best, poorly applicable for these data.

Finally, a set of challenging data used as a test case in the EQUIVALENT CIRCUIT fitting program [3] was further investigated by the present methods. We thank van Hassel of the University of Twente for providing it [33]. The zirconia material was measured at $500^{\circ} \mathrm{C}$. Fig. 8 shows the frequency response and complex-plane shape for these data. An excellent DRT fit was obtained with $M=8$ and $R_{\infty}$ free, and it
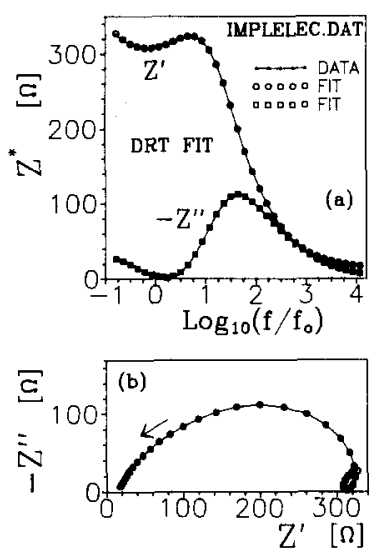

Fig. 8. (a) Log-log frequency response of the complex-conjugate impedance components of iron-implanted, cubic yttria-stabilized zirconia with a porous sputtered gold electrode. (b) The complex-plane response associated with the data of (a). The open symbols show DRT fit results with $M=8$.

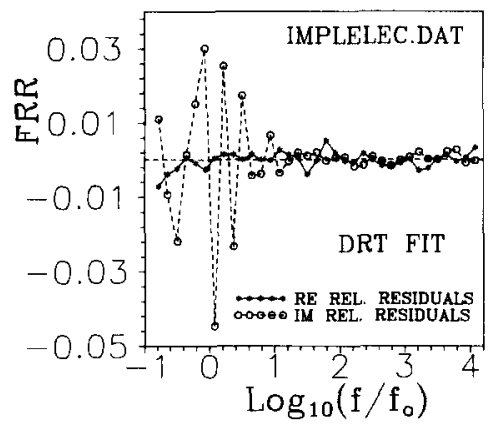

Fig. 9. Fit relative residuals for the DRT fit of the data of fig. 7.

led to $S_{\mathrm{F}}=0.010$. Fig. 9 presents the relative residual results for this fit. We see that they are satisfactorily small except in the neighborhood of $1 \mathrm{~Hz}$ where $-Z^{\prime \prime}$ is very small. Although this causes the relative residuals to be correspondingly magnified, the actual residuals in this region are small. Note that outside this region these results show no trace of the linear trends of the curves of Fig. 7, confirming the absence of significant time variation.

Fig. 10 shows the frequency response on a log-log scale and compares DRT fit results with those obtained by KK integration. Within the data range, both approaches yield good agreement with the data, but KKR testing requires somewhat subjective choices for the necessary high- and low-frequency extrapolated behaviors. The long-dash curves show that again the automatic DRT extrapolations differ somewhat from 


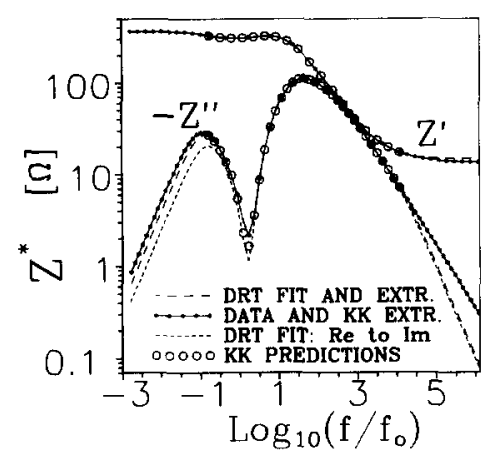

Fig. 10. $\log -\log$ frequency response for various different fits of the data of Fig. 8.

the present KK ones. It was found, however, that with $M=14$, DRT fitting of the full data used for KKRtesting yielded an excellent fit with $S_{\mathrm{F}}=0.0051$.

Thus far, we have dealt only with CNLS DRT fitting of both real and imaginary data components simultaneously. But the DRT method can also be used to predict real-part response from imaginary part and vice versa, just as the KKR do. Fig. 10 includes the results of one such prediction: a real-to-imaginary transformation. Here the real part of the original data was first very closely fitted by the DRT approach. Then the resulting parameter estimates (the fitting model) were used to calculate the full complex response over the entire frequency range, including the KK extrapolation-region frequencies. The imaginary part of this complex response is that plotted in Fig. 10 with short dashes. We see that it begins to deviate more and more from the data as the frequency decreases.

The lower-frequency response of the original $-Z^{\prime \prime}$ data is shown on an expanded linear scale in Fig. 11. This graph also shows the results of an imaginary-part fit and of the imaginary-part of a full complex fit. It is evident that even on this large scale, both results agree closely with the original data. But we also see how much the real-to-imaginary fit deviates from correct response at the low frequencies. Clearly this result is not fully satisfactory as a substitute for one of the KK transforms. By contrast, the corresponding imaginary-to-real estimate (not shown) is here an entirely adequate replacement for the other KK transform.

Finally, however, if should be mentioned that if the real-to-imaginary DRT procedure is carried out here

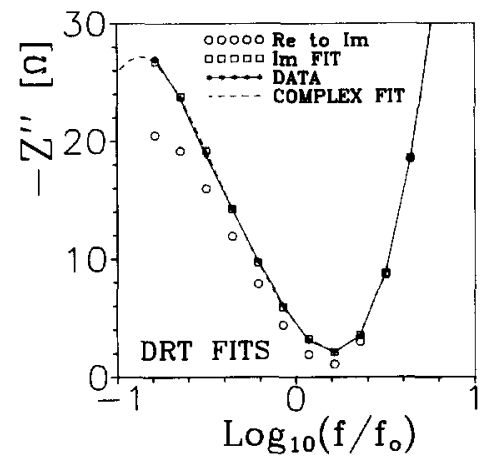

Fig. 11. Plot of $-Z^{\prime \prime}$ versus $\log$ frequency over the low frequency region for various types of DRT fits.

using not just the original data set but that plus the KK extrapolated points, one finds that over the original data range the predicted imaginary component then agrees very closely with the data: a fully successful transformation. Thus, we see that for the estimation of individual components by the DRT approach, there will be instances where one should augment the data with an extrapolated region, just as is necessary for the KKR. For the present data, where $-Z$ " exhibits a constant $\log -\log$ slope at high frequencies, individual-component transformation/estimation including such extrapolated response is clearly superior to fitting without such extrapolation.

An important advantage of the DRT method is that it can be applied directly to the measured data without the uncertain data extrapolation generally required by the KK-transform method. But because several fits with increasing $M$ may be needed for appreciable $M$ values, this approach may not be faster than optimized KK analysis, such as that now incorporated in the EQUIVALENT CIRCUIT program [3].

\section{The estimation of distributions of relaxation times and activation energies}

\subsection{Derivation and comparison of two discrete distributions}

Distributions may be either intrinsically continuous or discrete. Continuous ones are needed to describe DCE behavior exactly, but they may nevertheless be estimated in terms of discrete approximations 
to such continuous-distribution behavior [30]. Of course, if an actual distribution is discrete, no approximation is made in so treating it. Not only is fitting of the circuits of Fig. 4 to experimental data valuable for testing whether the data satisfy the KKR or not, as discussed above, but it provides methods of estimating the DRT 's and DAE's associated with the data in terms of discrete distribution weights. At the least, such estimation provides one with a value of the minimum number of time constants or relaxation times needed to describe the data adequately. At the most, it provides quantitative estimates of the distributions themselves, estimates that can often contribute appreciably towards improving the identification of the various physical processes present and increasing understanding of them.

Now on specializing Eq. (3) to the conductive-system impedance level and temporarily ignoring any contribution from dielectric effects, we can write

$Z(\omega)=Z_{\mathrm{C}}(\omega)=R_{\infty}+\Delta R I_{\mathrm{C}}(\omega)$,

where $\Delta R \equiv\left(R_{0}-R_{\infty}\right)$ and $Z^{\prime}(0) \equiv R_{0}$ (to be distinguished from the $R_{0}$ in Fig. 4). If $I_{\mathrm{C}}$ represents a single dispersion associated with a continuous DRT, we can rewrite the usual dielectric-system DRT expression $[8,20,34]$ to describe conductive-system response arising from a conductive-system continuous DRT, $I_{\mathrm{CG}}$, obtaining

$I_{C G}(\omega) \equiv \int_{0}^{\infty}[G(\tau) /(1+\mathrm{j} \omega \tau)] \mathrm{d} \tau$,

where the DRT $G(\tau)$ has the dimension of $\tau^{-1}$, and normalization requires that $I_{\mathrm{CG}}(0)=1$. A conductive-system dispersion relation of this kind was proposed as early as 1956 [8].

For a discrete DRT, we write $G(\tau)$ as a sum of delta functions,

$G(\tau)=\sum_{m=1}^{M} g_{m} \delta\left(\tau-\tau_{m}\right)$,

where the $g_{m}$ weight coefficients are dimensionless. Since measured data are always discrete, consider $N$ frequency values. Then Eq. (12) becomes, for each $\omega_{n}(1 \leqslant n \leqslant N)$,

$I_{\mathrm{C}_{\mathrm{B}}}\left(\omega_{n}\right)=\sum_{m=1}^{M} g_{m} /\left(1+\mathrm{j} \omega_{n} \tau_{m}\right)$, and $I_{\mathrm{Cg}}(0)=1$. The discrete DRT is then defined by the $g_{m}, \tau_{m}$ values.

Consider now the response of $M R C$ 's pairs in series, as in the top part of Fig. 4. Their impedance is

$Z_{\mathrm{Mg}}\left(\omega_{n}\right)=\sum_{m=1}^{M} R_{m} /\left(1+\mathrm{j} \omega_{n} R_{m} C_{m}\right)$,

where we may write $\tau_{m} \equiv R_{m} C_{m}$, and $Z_{\mathrm{Mg}}$ is not generally the full impedance of the system, as discussed below. Note that the $R_{0}$ in Fig. 4 is not $Z^{\prime}(0)$ but may be defined as $R_{\infty}$ when this quantity needs to be taken non-zero. Now substitution of Eq. (14) in Eq. (11) and comparison with Eq. (15) shows that

$g_{m}=R_{m} / \Delta R$,

and $\sum_{m=1}^{M} R_{m}=\Delta R$. Thus, fitting estimates of $R_{m}$ and $C_{m}$ (or $R_{m}$ and $\tau_{m}$ ) fully define the discrete DRT.

Next define $Y_{\mathbf{M g}_{\mathrm{g}}}\left(\omega_{n}\right) \equiv\left[Z_{\mathbf{M g}_{\mathrm{g}}}\left(\omega_{n}\right)\right]^{-1}$. It turns out that $Y_{\mathrm{Mg}}(\infty)$ is non-zero even when $R_{\infty}=0$, and it is then given by $\sum_{m=1}^{M}\left(R_{m} C_{m}^{2}\right)^{-1} /\left[\sum_{m=1}^{M} C_{m}^{-1}\right]^{2}$. But when $R_{\infty} \neq 0, Z_{\mathrm{M}}\left(\omega_{m}\right) \equiv R_{\infty}+Z_{\mathrm{Mg}}\left(\omega_{n}\right)$, and the corresponding $Y_{M}(\infty)=\left(R_{\infty}\right)^{-1}$, quite a different result.

The modulus response following from Eq. (15) is

$$
\begin{aligned}
& M_{\mathrm{Mg}_{\mathrm{g}}}\left(\omega_{n}\right)=\mathrm{j} \omega_{n} C_{\mathrm{V}} Z_{\mathrm{Mg}}\left(\omega_{n}\right) \\
& \quad=C_{\mathrm{V}} \Delta R \sum_{m=1}^{M} \mathrm{j} \omega_{n} g_{m} /\left(1+\mathrm{j} \omega_{n} \tau_{m}\right),
\end{aligned}
$$

and its $\omega_{n} \rightarrow \infty$ limit is $C_{\mathrm{v}} \Delta R \sum_{m=1}^{M} g_{m} / \tau_{m} \equiv$ $C_{\mathrm{V}} \Delta R\left\langle\tau^{-1}\right\rangle$. In terms of the circuit parameters, however, this limit is just $M_{\mathrm{Mg}}(\infty)=$ $C_{\mathrm{V}} \sum_{m=1}^{M}\left(C_{m}\right)^{-1} \equiv C_{\mathrm{V}} / C_{\mathrm{C} \infty} \equiv 1 / \epsilon_{\mathrm{C} \infty}$, where $C_{\mathrm{C} \infty}$ (or $\epsilon_{\mathrm{C} \infty}$ ) is the high-frequency-limiting capacitance (or dielectric constant increment) associated directly with conductive-system response.

Thus far, we have ignored any contribution from dielectric response of the bulk material. But any real material, even in the absence of dielectric- or conductive-system dispersion, has a high-frequency-limiting dielectric constant, $\epsilon_{\mathrm{D} \infty}$, which is present whether or not there is any conduction. But how do $\epsilon_{\mathrm{D}_{\infty}}$ and $\epsilon_{\mathrm{C} \infty}$ combine to yield a total high frequency limiting dielectric constant? Suppose in the top circuit of Fig. 4 we set $R_{\mathrm{s}}=0, R_{\mathrm{D}}=\infty$, and $C_{\mathrm{D}}=0$, and take $C_{\mathrm{P}}=$ $C_{\mathrm{v}} \epsilon_{\mathrm{D} \infty} \equiv C_{\mathrm{D} \infty}$, the high-frequency-limiting dielectricsystem capacitance. Then, the total circuit consists of $\left[R_{\infty}+\Delta R I_{\mathrm{C}}(\omega)\right]$ in parallel with the effects of $C_{\mathrm{D}_{\infty}}$. Now let us formally define $M_{\mathrm{C}}(\infty) \equiv M_{\mathrm{C} \infty}=1 / \epsilon_{\mathrm{C} \infty}$, 
as the $\omega \rightarrow \infty$ limit of $i \omega C_{\mathrm{V}} \Delta R I_{\mathrm{C}}(\omega)$. Physical realizability requires that this quantity, whether derived from a discrete or a continuous approach, must be neither zero nor infinity, in agreement with the discrete-distribution result above. But note that for certain empirical response functions, such as that of Davidson and Cole, proper results in the $\omega \rightarrow \infty$ limit require modification of the response at large but finite frequencies to ensure physical realizability [25]. Now we can write the total $M(\omega)$ of the present circuit as

$$
\begin{gathered}
M(\omega)=\mathrm{j} \omega C_{\mathrm{v}}\left[R_{\infty}+\Delta R I_{\mathrm{C}}(\omega)\right] / \\
{\left[1+\mathrm{j} \omega C_{\mathrm{D} \infty}\left\{R_{\infty}+\Delta R I_{\mathrm{C}}(\omega)\right\}\right] .}
\end{gathered}
$$

It follows that $M(0)=0$ and $Z(0)=R_{0}$, as it should. Now for the $\omega \rightarrow \infty$ limit, Eq. (18) leads to

$$
\begin{gathered}
M(\infty) \rightarrow\left[M_{\mathrm{C} \infty}+\mathrm{j} \omega C_{\mathrm{v}} R_{\infty}\right] / \\
{\left[1+\epsilon_{\mathrm{D} \infty} M_{\mathrm{C} \infty}+\mathrm{j} \omega C_{\mathrm{D} \infty} R_{\infty}\right],}
\end{gathered}
$$

where the $\omega \rightarrow \infty$ limit has been temporarily deferred for two terms. If $R_{\infty} \equiv 0$, then we find $M(\infty)=1 /$ $\epsilon_{\infty} \equiv 1 /\left(\epsilon_{\mathrm{D} \infty}+\epsilon_{\mathrm{C} \infty}\right)$ on setting $M_{\mathrm{C} \infty}=1 / \epsilon_{\mathrm{C} \infty}$. On the other hand, when $R_{\infty} \neq 0, M(\infty)=1 / \epsilon_{\infty} \equiv 1 / \epsilon_{\mathrm{D} \infty}$. In this latter case, we see that although $M_{\mathrm{C}_{\infty}}$ is non-zero, the presence of $R_{\infty}$ keeps it from contributing to the overall high-frequency-limiting total dielectric constant of the system.

Now it proves useful to consider another discrete DRT which is related to the one above. Instead of Eq. (14), write [8]

$I_{C h}\left(\omega_{n}\right)=\sum_{m=1}^{M} \tau_{m} h_{m} /\left(1+\mathrm{j} \omega_{n} \tau_{m}\right)$,

with

$I_{\mathrm{C} h}(0)=\sum_{m=1}^{M} \tau_{m} h_{m} \equiv\langle\tau\rangle_{h}=1$.

Now, in analogy to Eq. (15), write

$Z_{\mathrm{M} h}\left(\omega_{n}\right)=\sum_{m=1}^{M} \tau_{m} H_{m} /\left(1+\mathrm{j} \omega_{n} \tau_{m}\right)$,

Because $\sum_{m=1}^{M} \tau_{m} H_{m}$ must equal $\Delta R$, it follows that

$h_{m}=H_{m} / \Delta R$.

Since $h_{m}$ is not normalized to a sum of unity, finally let $p_{m} \equiv h_{m} / \sum_{m=1}^{M} h_{m}$. Here the free fitting parame- ters are $H_{m}$ and $\tau_{m}$, and their converged fit values may be used to calculate $\Delta R, h_{m}$, and $p_{m}$, with the new normalized discrete distribution finally defined by $p_{m}$ and $\tau_{m}$.

The dimensionless modulus response associated with dispersion following from Eq. (21) can now be written

$$
\begin{aligned}
& M_{\mathrm{Mp}}\left(\omega_{n}\right)=\left[C_{\mathrm{V}} \Delta R \sum_{m=1}^{M} h_{m}\right] \sum_{m=1}^{M} \mathrm{j} \omega_{n} \tau_{m} p_{m} / \\
& \left(1+\mathrm{j} \omega_{n} \tau_{m}\right)
\end{aligned}
$$

whose $\omega_{n} \rightarrow \infty$ limit is just

$M_{\mathrm{Mp}}(\infty)=C_{\mathrm{V}} \Delta R \sum_{m=1}^{M} h_{m}$.

Although the relations

$\sum_{m=1}^{M} g_{m}=\sum_{m=1}^{M} \tau_{m} h_{m}=\sum_{m=1}^{M} h_{m} \sum_{m=1}^{M} \tau_{m} p_{m}$

seem to impose only global connections between $g_{m}$, $h_{m}$, and $p_{m}$, it turns out that when these quantities are determined by CNLS fitting, then for all $m, h_{m}=g_{m} /$ $\tau_{m}$, with the same $\tau_{m}$ estimates obtained for both approaches. Thus, the quantity $\sum_{m=1}^{M} h_{m}=\left\langle\tau^{-1}\right\rangle$, and the results for the $g$-distribution given above apply here as well. Therefore,

$M_{\mathrm{M} p}(\infty)=M_{\mathrm{Mg}}(\infty)=C_{\mathrm{V}} \Delta R\left\langle\tau^{-1}\right\rangle \equiv 1 / \epsilon_{C \infty}$.

This expression properly relates the low-frequency limiting part of the distributed impedance, $\Delta R, a$ purely conductive-system quantity, to a dielectric constant increment associated with mobile charges, not with dipole rotation, electronic polarization, and vibration effects, and proper results for the total $M(\infty)$ are found when the dielectric-system-capacitance, $C_{\mathrm{D} \infty}$, and the limiting resistance $R_{\infty}$ are included in the response, as discussed above.

As we shall see, the distribution defined by values of $p_{m}$ and $\tau_{m}$ is useful as well as that associated with $g_{m}$ and $\tau_{m}$. Further, the above results demonstrate that one need not even use the fitting of Eq. (21) to obtain $p_{m}$ and $\tau_{m}$ estimates. Instead, once $g_{m}$ and $\tau_{m}$ values are available, one can immediately calculate $p_{m}$ values without further fitting. It is worth mentioning, however, that the relative standard deviation estimates for $p_{m}$ values obtained by direct fitting have been found to be somewhat smaller than those of $g_{m}$. 
Earlier work related to the result of Eq. (23) is discussed in the Appendix.

\subsection{Estimation of conducting-system distributions}

As discussed elsewhere [30], there are problems in the estimation of truly continuous distributions. The determination of such a distribution from discrete data is an inverse problem, a notoriously difficult task and one which is both ill-posed and ill-conditioned mathematically. It is worth emphasizing that this problem is present no matter how well the fitting model fits the data. For example, for the worst of the distribution estimates obtained below with exact data, fitting with $M=17$ yielded a value for $S_{\mathrm{F}}$ of only $6.7 \times 10^{-4}$, a very close fit of the original data. If, alternatively, the underlying distribution involves only a finite number of discrete simple relaxation processes, their estimation is not an ill-posed problem, and one can expect to obtain estimates limited only by the accuracy of the data using the present discretefunction approximation [30].

In spite of the above intrinsic difficulty with continuous-distribution estimation, it is of interest to evaluate the adequacy of such estimation for optimum conditions, i.e. using essentially exact data. To do so, one needs to generate exact frequency response data from a known continuous distribution. For convenience, we shall use the EDAE of Eqs. (8) to (10) to generate such data. Fig. 12 compares estimated results to the original exact EDAE's for the $\mathscr{E}_{\mathrm{H}}=10, \phi=0.7$ choices. The fitting results are for

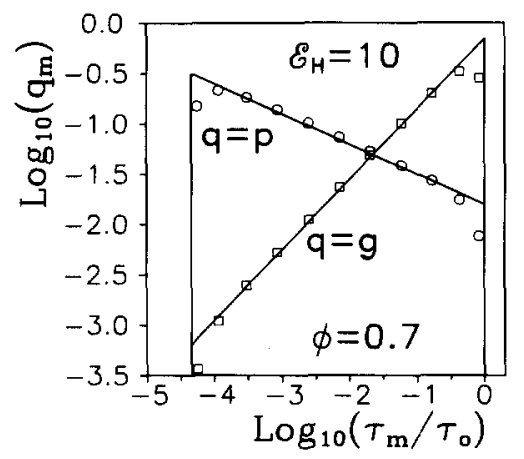

Fig. 12. Log-log plot of exact EDAE continuous distribution (solid lines) and discrete DRT weight estimates of each distribution for $\phi=\phi_{g}=0.7$ ( $q=g$, open squares) and $\phi=\phi_{p}=-0.3$ ( $q=p$, open circles). Here $\mathscr{G}_{\mathrm{H}}=10, \tau_{0}=\tau_{\mathrm{H}}$, and $M=11$.
$M=11$. Here the exact distributions were calculated using Eq. (6) for $\phi=0.7$ and Eq. (7) for $\phi=-0.3$. Let us denote the usual $\phi$ associated with the $g_{m}$ distribution as $\phi=\phi_{g}$ and that with the $p_{m}$ distribution as $\phi_{p}$. Then take $q_{m}$ as either $g_{m}$ or $p_{m}$.

The $q=g$ distribution is the same as the one presented at the top of Fig. 1 when the scales are changed appropriately. We see that the discrete $g_{m}$ results, denoted by open squares (Fig. 12) agree surprisingly well with the exact continuous distribution except near the sharp cutoffs at the ends of the distribution. Certainly, they delineate the shape of the actual distribution surprisingly well.

Since $p_{m}$ is proportional to $g_{m} / \tau_{m}$, the continuous EDAE which is associated with it must be proportional to that associated with $g_{m}$ divided by $\tau$ or, equivalently, $\exp (\mathscr{E})$. It immediately follows that the latter distribution is the same as the first but with $\phi_{p}$ replaced by $\phi_{g} \equiv \phi_{p}+1$. So when $\phi_{g}=0.7, \phi_{p}=-0.3$. Comparison of the exact distribution and its discrete-point estimates (open circles, Fig. 12) again shows excellent agreement except at the ends of the distribution. The slopes of the two middle-region solid lines in the figure are exactly equal to the values of $\phi_{g}$ and $\phi_{p}$. Thus, when a region of constant log-log slope appears, one can immediately obtain an estimate of $\phi$. Further, since $\log _{10}\left(\tau_{\max } / \tau_{\min }\right)$ is related to $\mathscr{E}_{\mathrm{H}}$ as discussed in Section 2, one can also obtain an estimate of $\mathscr{E}_{\mathbf{H}}$. Although the present results were obtained using conducting-system synthetic data to obtain estimates of conducting-system DRT's, if one alternatively takes the same data as representing dielectric-system response, LEVM allows this data set to be analyzed to yield a dielectric-system DRT, one which is exactly the same in normalized form as the conducting-system one. Thus, the present results apply to both kinds of response.

Fig. 13 shows a comparison of the exact exponential distribution for $\mathscr{E}_{\mathbf{H}}=20$ and $\phi=1$ with the discrete-distribution $g_{m}$ estimates. Here, although the $M=17$ estimates show the correct slope, their middle-region magnitudes are somewhat too large. The discrepancy is made clearer by the results of Fig. 14 for $p_{m}$. Not only are deviations from a zero-slope line easier to observe than those from a line with appreciable slope, but here an expanded-scale linear axis is used for greater resolution. The open circles mark the position of the $p_{m}$ points, and the uncertainty rect- 


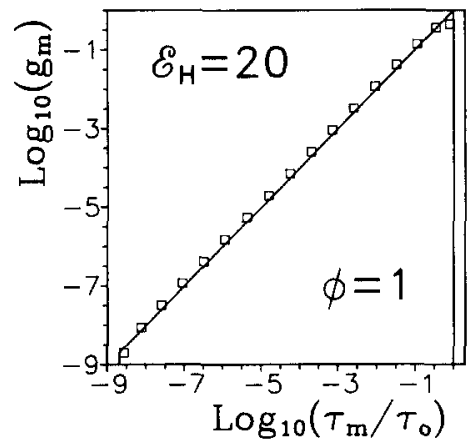

Fig. 13. Log-log plot of exact and estimated EDAE distributions for $\mathscr{E}_{\mathrm{H}}=20, \phi=1$ and $\tau_{0}=\tau_{\mathrm{H}}$.

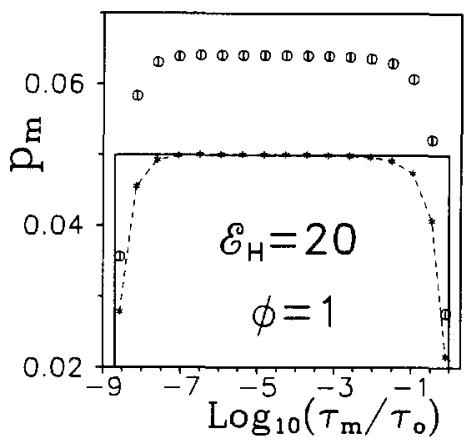

Fig. 14. Linear-log plot of exact and estimated EDAE distributions showing the $p_{m}$ response associated with the choices $\phi_{g}=1$ and $\phi_{p}=\phi_{g}-1=0$ for $\varepsilon_{\mathrm{H}}=20$. Here the small rectangles within the open circles are defined by \pm 1 estimated standard deviation lines for the simultaneous uncertainties in both $p_{m}$ and $\tau_{m}$. The dashed line is a scale-corrected estimate of the distribution (see text). Here $\tau_{0}=\tau_{\mathrm{H}}$.

angles, which mostly fall within the circles, are constructed with lines at \pm one estimated standard deviation of $p_{m}$ and of $\tau_{m}$ from the circle centers. They are too small to resolve on the preceding $\log -\log$ graphs and so are not included there.

Now let $p_{m_{e}}$ denote the estimated flat-top value of $p_{m}$. It turns out that although $p_{m_{e}}$ is virtually independent of the number and spacing of the original data points if a sufficient number are included to cover the main region of response, $p_{m_{e}}$ does depend on $M$. An improved estimate of the central $p_{m}$ values, $p_{m_{\mathrm{c}}}$, is given by $p_{m_{\mathrm{c}}}=M p_{m_{\mathrm{e}}} /\left(1.013 \mathscr{E}_{\mathrm{H}}+1.51\right)$. It is useful over a considerable range of values of $M$ and $\mathscr{E}_{\mathrm{H}}$. The result of applying this transformation to the present data is shown in Fig. 14 by the dashed line connecting the resulting $p_{m_{c}}$ points.

But why should such a correction be necessary? It is, in fact, unnecessary when a discrete distribution is estimated using a discrete-function approach, such as that employed here. But the present discrepancy is associated with the estimation of a continuous distribution by a discrete-line one. Although the area under a continuous distribution, such as a continuousfunction choice for the $G(\tau)$ of Eq. (12) or the specific distributions of Fig. 12, is normalized to unity, the corresponding discrete-function sum, $\sum_{m=1}^{M} p_{m}$, which is here also required to be unity to yield $Z_{\mathrm{M} h}(0)=\Delta R$ in Eq. (21), is not a good approximation to the continuous-distribution integral. But the above correction adjusts in considerable measure for the difference.

As pointed out recently by Morgan and Lesmes [33], for comparison of a continuous-function approximation to a continuous distribution, it is appropriate to renormalize the inversion coefficients so that the area under the curve defined by them is the same as that of the continuous distribution to which they are being compared. Such renormalization is also appropriate if we reinterpret the present discrete-function-approach estimated line-spectra coefficients, $q_{m}$ and $\tau_{m}$, as defining instead a continuous distribution. In its simplest form, such renormalization is very similar to the above correction, but appreciably greater accuracy is obtained if DRT integrals, such as that of Eq. (12), are approximated by cubic spline fitting and integration [17,30].

Finally, can anything interesting be found from estimating the distributions associated with the experimental Implelec data (Fig. 8) used above for KKR and DRT analyses? Figs. 15 and 16 show such results with $M=8$. Larger values of $M$ led to great uncertainties in some of the parameter estimates, thus indicating that the accuracy of the data supports only a maximum value of $M=8$. The value of $S_{\mathrm{F}}$ found from the fitting was about 0.0103 using the sixteen free parameters associated with the distribution and a bulk resistance in series. The estimated value of this resistance was $14.82 \pm 0.25 \Omega$, where 0.25 is the estimated standard deviation.

The figures show that the uncertainties of many of the estimated distribution quantities are quite appre- 


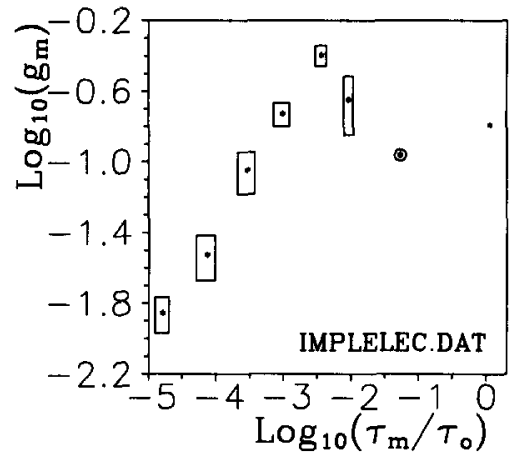

Fig. 15. $\mathrm{Log}$ - $\log$ plot of discrete DRT $g_{m}$-estimates of the distribution associated with the Implelec data of Fig. 8. The rectangles are formed as described in the caption to Fig. 14 and the point enclosed by a circle involves a negative value of $g_{m}$.

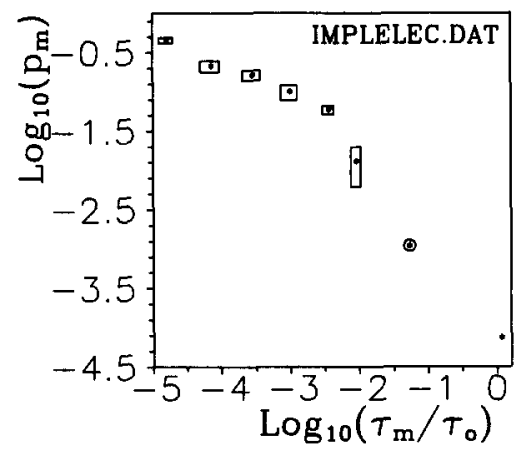

Fig. 16. Log-log plot of discrete DRT $p_{m}$-estimates of the distribution associated with the data of Fig. 8. Definitions are the same as those listed in the caption of Fig. 15.

ciable here, and thus the standard-deviation rectangles show clearly on these $\log$-log plots for most of the points. But the uncertainties are too small to be visible for the two larger- $\tau$ points. The largest- $\tau$ point indicates the presence of a well-determined discrete time constant involving a resistance of about $55.6 \pm 0.6 \Omega$ in parallel with a capacitance of about $0.0204 \pm 0.0002 \mathrm{~F}$. The second point from the right on both plots, identified by an enclosing circle, is different from the others. The fitting yielded one welldetermined $R C$ pair for which both the $R_{m}$ and the $C_{m}$ were negative [35], but their product, $\tau_{m}$, was positive. Thus the $g_{m}$ and $p_{m}$ values for this point are negative.

These results suggest that the well-defined two points on the right represent discrete contributions to the overall DRT and that at least the five points on the left are associated with a continuous distribution. To the degree that meaningful straight lines can be drawn through these lower- $\tau$ five points, one can obtain log-log slope estimates of about 0.62 and -0.38 for these regions. The data are clearly insufficiently accurate to allow one to conclude conclusively that an EDAE, such as those discussed earlier, is present, but certainly this seems reasonably likely. If we accept this possibility, then the maximum activation energy $E_{\mathrm{H}}$ can be estimated as $k_{\mathrm{B}} T \ln \left(\tau_{0} / \tau_{\min }\right)$. On using the measurement temperature and assuming on the basis of the fit results that $\tau_{\min } / \tau_{0} \simeq 10^{-5}$, we obtain $\mathscr{E}_{\mathrm{H}} \simeq 11.5$ and so $E_{\mathrm{H}} \simeq 0.77 \mathrm{eV}$.

Essentially these same data have been fitted [3] by equivalent circuits using 9 to 11 free parameters and one or two distributed circuit elements (CPE's). For the present work, the data have again been fitted to one of these circuits and using the same weighting (FPWT - see $[4,29]$ ) as used in the DRT fitting above. The fit led to an $S_{\mathrm{F}}$ value of 0.0175 and to a series resistance estimate of $13.97 \pm 0.18 \Omega$. In addition, it led to values of a resistance and capacitance in parallel of $55.5 \pm 1.4 \Omega$ and $0.0235 \pm 0.0004 \mathrm{~F}$, respectively, very similar to the corresponding DRT values. A resistance and parallel inductance were used in this equivalent circuit to represent the inductive loop represented above by a negative resistance and negative capacitance [35]. Finally, a CPE was included in the equivalent circuit, and fitting gave an estimate of its exponent of about 0.73 , of the same order of magnitude as the log-log slope of about 0.62 found from the DRT fitting. These results show that had no equivalent circuit been available at the start of fitting, results from the DRT-fitting approach would have suggested some needed elements in such an equivalent circuit and even suggested useful initial values for some of the free parameters.

\section{Appendix 1}

A widely-used expression overtly similar to Eq. (23) of the text was presented many years ago for distributed conductive-system response by Macedo et al. [36], but it differs in subtle but important ways from the present results. Although these authors did not explicitly introduce a separate high-frequencylimiting capacitance or resistance in the way dis- 
cussed above, they did consider a modulus function representing the total response of the measured system and took $M(\infty) \equiv M_{\mathrm{s}}=1 / \epsilon_{\mathrm{s}}$, with $\epsilon_{\mathrm{s}}$ defined as containing "all ordinary contributions to the relative permittivity except those connected with the long range ionic diffusion process" [36]. Thus, $\epsilon_{\mathrm{s}}=\epsilon_{\mathrm{D} 0}=$ $\epsilon_{\mathrm{D} \infty}$ in the absence of dielectric dispersion.

If we replace the $M_{M p}(\infty)$ quantity in Eqs. (23) through (25) by the Macedo et al. $M_{\mathrm{s}}$, the present result for $M_{M p}\left(\omega_{n}\right)$ and theirs are identical in form when theirs is transformed from a continuous to a discrete distribution. But, as shown by Eq. (25), this replacement is equivalent to changing from $1 / \epsilon_{\mathrm{C}_{\infty}}$ to $1 / \epsilon_{D_{\infty}}$, quite a different quantity. Thus the essence of the difference between the Macedo et al. approach and the present one arises from their failure to distinguish between these two quantities, one a conducting-system result and the other only relevant for a dielectric system.

Consider first the consequence of this difference at the modulus level for the simplest case: $M=1$, one which here involves no conductive-system dispersion but, as follows from Eq. (15), only a single Debye relaxation involving the related conductive-system parameters $R_{1}$ and $C_{1}$ and the relaxation time $\tau_{1} \equiv R_{1} C_{1}$. When $R_{\infty}=0$, so $\Delta R=Z^{\prime}(0) \equiv R_{0}=R_{1}$, the response involves $R_{1}$ in parallel with the parallel combination of $C_{D_{\infty}}$ and $C_{1}$. Thus, as in the work of Section 4.1 above, $\epsilon_{\infty}=\left(\epsilon_{\mathrm{D} \infty_{\infty}}+\epsilon_{\mathrm{C}_{\infty}}\right)$, where here $\epsilon_{\mathrm{C} \infty}=C_{1} / C_{\mathrm{v}}$. Although physical realizability precludes the possibility of $R_{\infty}$ ever being identically zero [25], $R_{\infty}$ will often be so small that there will be an extensive frequency region where the above expression for $\epsilon_{\infty}$ holds accurately. Eventually, however, this quantity must approach $\epsilon_{\mathrm{D} \infty}$.

In contrast, the Macedo et al. approach in the absence of conductive-system dispersion may be interpreted, for $M=1$, as involving a conductive-system dc resistance $R_{0}=R_{1}$, a dielectric-system capacitance $C_{\mathrm{D} \infty}$, and the dielectric relaxation time $\tau_{\sigma} \equiv R_{0} C_{\mathrm{D} \infty}=$ $\epsilon_{\mathrm{v}} \epsilon_{\mathrm{Doo}} / \sigma_{0}$, with no separate $C_{1}$ term. Thus, their $M(\infty)=1 / \epsilon_{s}=1 / \epsilon_{\infty}=1 / \epsilon_{\mathrm{D} \infty}$, and no conductivesystem capacitative contribution to $\epsilon_{\infty}$ is present in any frequency range.

The difference between the predictions of the two approaches is particularly interesting and important at the impedance level for arbitrary $M$. Ignore $R_{\infty}$; then the present approach (e.g., Eqs. (11), (15)) yields $Z^{\prime}(0)=\Delta R$ as it must. But the Macedo et al. analysis leads to

$$
\begin{gathered}
Z^{\prime}(0)=\left(M_{\mathrm{s}} / C_{\mathrm{V}}\right) \sum_{m=1}^{M} \tau_{m} p_{m}=\left(M_{\mathrm{s}} / C_{\mathrm{V}}\right)\left[\sum_{m=1}^{M} h_{m}\right]^{-1} \\
=\left(\epsilon_{\mathrm{D} \infty} C_{\mathrm{V}}\right)^{-1} /\left\langle\tau^{-1}\right\rangle=\left(\epsilon_{\mathrm{C} \infty} / \epsilon_{\mathrm{D} \infty}\right) \Delta R, \quad \text { (A1) }
\end{gathered}
$$

on using the right parts of Eq. (25). Since $\epsilon_{\mathrm{C}_{\infty}}$ and $\epsilon_{\mathrm{D} \infty}$ are different quantities, this result is clearly incorrect and again demonstrates the inconsistency of the Macedo et al. approach, one which should be replaced by the present one.

\section{Definition of acronyms}

CPE Constant-phase circuit element

CNLS Complex nonlinear least squares

DAE Distribution of activation energies

DCE Distributed circuit element

DRT Distribution of relaxation times

EDAE Exponential distribution of activation energies

EIS Electrochemical impedance spectroscopy

IS Impedance or immittance spectroscopy

KK Kronig-Kramers

KKR Kronig-Kramers integral relations

LEVM Levenberg-Marquardt-Macdonald CNLS program

\section{References}

[1] J.R. Macdonald, ed., Impedance Spectroscopy Emphasizing Solid Materials and Systems (WileyInterscience, New York, 1987).

[2] J.R. Macdonald, J. Schoonman and A.P. Lehnen, J. Electronanal. Chem. 131 (1982) 77.

[3] B.A. Boukamp, Solid State Ionics 18/19 (1986) 136; 20 (1986) 31 .

[4] J.R. Macdonald and L.D. Potter, Jr., Solid State Ionics 23 (1987) 61.

[5] J.R. Macdonald, Electrochim. Acta 35 (1990) 1483.

[6] R. de L. Kronig, J. Opt. Soc. Am. 12 (1926) 547.

[7] H.A. Kramers, Atti Gongr. dei Fisici, Como (1927) 545; Physik. Z. 30 (1929) 52.

[8] J.R. Macdonald and M.K. Brachman, Rev. Mod. Phys. 28 (1956) 393.

[9] D.D. Macdonald and M. Urquidi-Macdonald, J. Electrochem. Soc. 132 (1985) 2316. 
[10] M. Urquidi-Macdonald, S. Real and D.D. Macdonald, J. Electrochem. Soc. 133 (1986) 2018.

[11] D.D. Macdonald and M. Urquidi-Macdonald, Corrosion 45 (1989) 327.

[12] M. Urquidi-Macdonald, S. Real and D.D. Macdonald, Electrochim. Acta 35 (1990) 1559.

[13] J.M. Esteban and M.E. Orazem, J. Electrochem. Soc. 138 (1991) 67

[14] M.E. Orazem, J.M. Esteban and O.C. Moghissi, Corrosion 47 (1991) 248.

[15] J.R. Macdonald, Electrochim. Acta 38 (1993) 1883.

[16] J. Callaway, Quantum Theory of the Solid State, 2nd. Ed. (Harcourt-Brace-Jovanovich, Boston, 1991).

[17] B.A. Boukamp, Solid State Ionics 62 (1993) 131.

[18] J.R. Macdonald, J. Appl. Phys. 58 (1985) 1955.

[19] J.R. Macdonald and J.C. Wang, Solid State Ionics 60 (1993) 319.

[20] C.J.F. Bottcher and P. Bordewijk, Theory of Electric Polarization, Vol. II (Elsevier, Amsterdam, 1978). See p. 58 and earlier references listed.

[21] J.R. Macdonald, J. Appl. Phys. 61 (1987) 700. (See especially Fig. 5.)
[22] D.W. Davidson and R.H. Cole, J. Chem. Phys. 19 (1951) 1484.

[23] J.R. Macdonald, Phys. Rev. B 49 (1994-II) 9428.

[24] J.R. Macdonald, J. Electroanal. Chem., to be published.

[25] J.R. Macdonald, Solid State Ionics 25 (1987) 271.

[26] H.J. Woltring, Adv. Eng. Software 8 (1986) 104.

[27] A.S. Nowick, private communication.

[28] B. Gross, Mathematical Structure of the Theories of viscoelasticity, Actualites Scientifiques (Herman et Cie, Paris, 1953, 1967) p. 61.

[29] J.R. Macdonald, Manual, LEVM CNLS program.

[30] J.R. Macdonald, J. Chem. Phys., submitted for publication.

[31 ] P. Agarwal, M.E. Orazem and L.H. Garcia-Rubio, J. Electrochem. Soc. 139 (1992) 1917.

[32] M.E. Orazem, P. Agarwal, A.N. Jansen, P.T. Wojcik and L.H. Garcia-Rubio, Electrochim. Acta 38 (1993) 1903.

[33] Private communication.

[34] J.R. Macdonald, J. Appl. Phys. 75 (1994) 1059.

[35] D.R. Franceschetti and J.R. Macdonald, J. Electroanal. Chem. 82 (1977) 271. (See Table 2 and Fig. 4-d, 4-e).

[36] P.B. Macedo, J.H. Ambrus, C.T. Moynihan and R. Bose, Phys. Chem. Glasses 13 (1972) 17I. 